\title{
DETERMINACIÓN DEL TIEMPO MÁXIMO PARA RECUPERAR Y CRIOPRESERVAR ESPERMATOZOIDES OBTENIDOS DE LA COLA DEL EPIDÍDIMO EN CANINOS POST ORQUIECTOMÍA
}

\author{
Optimal Time for Recovery and Cryopreservation of Epididymal Canine \\ SPERM AfTer OrChiEctomy
}

Sandra Armas R. ${ }^{1}$, Víctor Fernández A.², María Vásquez C. ${ }^{3}$, Alexei Santiani A. ${ }^{1,4}$

\section{Resumen}

\begin{abstract}
El objetivo del estudio fue determinar el tiempo máximo para recuperar y criopreservar espermatozoides obtenidos de la cola del epidídimo de caninos post orquiectomía. Se obtuvo testículos de 20 caninos entre 1 y 8 años de edad. Los testículos se colocaron en cloruro de sodio al $0.9 \%$ y se almacenaron a $5{ }^{\circ} \mathrm{C}$ durante $0,24,48$ y 72 horas. Los espermatozoides fueron recuperados al cortar la cola del epidídimo en un dilutor en base a Tris-citrato-fructosa. Para el proceso de criopreservación, se añadió yema de huevo $(20 \%)$ y glicerol (5\%) a la muestra diluida (espermatozoides + dilutor). La nueva dilución fue envasada en pajillas de $0.5 \mathrm{ml}$ y sometidas a una curva de enfriamiento para luego ser colocadas en nitrógeno líquido. Se evaluó motilidad total, motilidad progresiva e integridad funcional de membrana. Previo a la criopreservación, la motilidad total varió significativamente después de 24 y 72 horas de almacenamiento a $5{ }^{\circ} \mathrm{C}(\mathrm{p}<0.05)$. Sin embargo, la motilidad total encontrada después del proceso de criopreservación sólo varió después de 72 horas de almacenamiento a $5^{\circ} \mathrm{C}(\mathrm{p}<0.05)$. Similar tendencia se observó para la motilidad progresiva. La integridad funcional de membrana plasmática, tanto antes como después del proceso de criopreservación, no sufrió cambios significativos entre los grupos. Los resultados demuestran que es posible recuperar y criopreservar espermatozoides epididimarios hasta después de 48 horas de la orquiectomía del animal.
\end{abstract}

Palabras clave: espermatozoide, epidídimo, canino, criopreservación de semen

\section{Abstract}

The aim of the study was to determine the maximum time to recover and cryopreserve spermatozoa from the tail of canine epididymis post orchiectomy. The testes were obtained through orchiectomy from 20 dogs aged 1 to 8 years. The testis were placed in sodium chloride $0.9 \%$ and stored at $5{ }^{\circ} \mathrm{C}$ for $0,24,48$ and 72 hours. Spermatozoa were recovered by cutting the tail of the epididymis in an extender based on Tris-citrate-fructose. For the

\footnotetext{
${ }^{1}$ Laboratorio de Reproducción Animal, ${ }^{2}$ Clínica de Animales Menores, ${ }^{3}$ Laboratorio de Fisiología Animal, Facultad de Medicina Veterinaria, Universidad Nacional Mayor de San Marcos, Lima

${ }^{4}$ E-mail: asantiani@hotmail.com
} 
cryopreservation process, egg yolk (20\%) and glycerol (5\%) was added to the diluted sample (sperm + dilutor). The new dilution was packaged in $0.5 \mathrm{ml}$ straws, which were subjected to a cooling curve and then placed in liquid nitrogen. Total motility, progressive motility and functional integrity of the membrane were evaluated. Before cryopreservation process, total motility after 24 and 72 hours of storage at $5{ }^{\circ} \mathrm{C}$ decreased $(\mathrm{p}<0.05)$. However, total motility found after cryopreservation process varied significantly only after 72 hours of storage at $5^{\circ} \mathrm{C}(\mathrm{p}<0.05)$. Similar trend was observed for progressive motility. The functional integrity of membrane, both before and after the cryopreservation process, did not suffer significant changes between groups. The results showed that it is possible to collect and cryopreserve epididymal sperm until 48 hours of orchiectomy.

Key words: sperm, epididymis, canine, semen cryopreservation

\section{INTRODUCCIÓN}

Actualmente, la criopreservación de semen canino es una herramienta importante para preservar espermatozoides de reproductores considerados como valiosos. Existen diversos métodos convencionales para la colección de semen; sin embargo, existen eventos impredecibles como accidentes, intoxicaciones o enfermedades, que súbitamente pueden desencadenar en la orquiectomía, muerte o eutanasia de perros de valor genético o sentimental. En estos casos, los propietarios no sólo deben afrontar la pérdida del animal, sino también la pérdida del material genético deseable. En ese sentido, es posible obtener espermatozoides después de la muerte del animal, los cuales son recuperados de la cola del epidídimo, con motilidad y capacidad fecundante (Yu et al., 2002).

En el epidídimo se realizan dos eventos importantes, la maduración y el almacenamiento espermático. La maduración o desarrollo progresivo de la capacidad fecundante de los espermatozoides ocurre en la cabeza y el cuerpo del epidídimo y el almacenamiento ocurre en la cola del epidídimo (Hafez, 2002). Es así, que la obtención de espermatozoides epididimarios, potencialmente fecundantes que se encuentran almacenados en la cola del epidídimo puede ser la única opción para preservar el material genético de un macho de alto valor genético, luego de su muerte u orquiectomía por motivos médicos (Filtanelli et al., 2007).

Se han realizado estudios sobre recuperación y criopreservación de espermatozoides epididimarios en bovinos (Amann y Griel, 1976), equinos (Bruemmer et al., 2002), ovinos (Kaabi et al., 2003), caprinos (Blash et al., 2000), y porcinos (Ikeda et al., 2002). En caninos, existen estudios sobre recuperación, refrigeración y criopreservación de espermatozoides obtenidos de la cola del epidídimo, especialmente el de Hewitt $e t$ al. (2001), quienes evaluaron variables espermáticas después de la orquiectomía; el de Yu y Leibo (2002) que estudiaron el efecto sobre la motilidad, integridad de membrana plasmática y acrosomal de espermatozoides epididimarios refrigerados; y el de Tittarelli et al. (2006), donde se evaluó la capacidad de supervivencia de los espermatozoides caninos mantenidos dentro de epidídimos refrigerados en solución salina isotónica estéril y Tris-yema de huevo.

No obstante, en los casos que se recupere espermatozoides de un macho recientemente muerto o sacrificado, es poco probable que en ese momento existan hembras en celo listas para ser inseminadas; por lo tanto, estos espermatozoides deberán ser criopreservados para poder mantenerlos por un periodo de tiempo indefinido hasta su utilización. En ese sentido, existe escasa infor- 
mación sobre congelación de espermatozoides recuperados de epidídimos mantenidos en refrigeración.

El presente estudio tuvo por objetivo evaluar el efecto del tiempo transcurrido entre la muerte $u$ orquiectomía de un perro hasta la recuperación de los espermatozoides del epidídimo, sobre la calidad espermática antes y después del proceso de criopreservación.

\section{Materiales y Métodos}

\section{Lugar de Estudio y Animales}

El estudio se realizó en el Laboratorio de Reproducción Animal de la Facultad de Medicina Veterinaria, Universidad Nacional Mayor de San Marcos (FMV-UNMSM), Lima, entre junio de 2008 a marzo de 2009. Se utilizó 20 caninos adultos (de raza y mestizos), entre 1 y 8 años de edad, aparentemente saludables, que fueron sometidos a orquiectomía. Los animales procedieron de los cursos de Cirugía Veterinaria y de Patología Veterinaria de la FMV-UNMSM, así como de campañas de esterilizaciones realizadas en varios distritos de Lima.

Los testículos escindidos fueron ligados en el cordón espermático e inmediatamente colocados en vasos precipitados (un par por cada vaso) conteniendo solución salina fisiológica ( $\mathrm{NaCl}$ al 0.9\%, pH: $5.4 \pm 0.2)$, a temperatura ambiente para evitar la desecación. Las muestras fueron transportadas a temperatura ambiente desde el sitio de recolección hasta el laboratorio en un plazo máximo de 2 horas.

\section{Metodología}

Se evaluó el efecto del tiempo de almacenamiento de espermatozoides post orquiectomía sobre la calidad espermática antes y después de la criopreservación. La recuperación de espermatozoides del epidídimo después de la orquiectomía se realizó a las 0 (Grupo 0), 24 (Grupo 24), 48 (Grupo 48) y 72 horas (Grupo 72).

Los testículos se distribuyen en forma aleatoria entre los cuatro grupos $(n=10)$, de tal forma que ningún testículo del mismo par fue asignado a un mismo grupo. Las muestras de los grupos 24,48 y 72 fueron mantenidos en refrigeración $\left(5{ }^{\circ} \mathrm{C}\right)$ desde la llegada al laboratorio hasta su procesamiento.

Recuperación de espermatozoides epididimarios

El testículo fresco o refrigerado, fue colocado en una placa Petri, donde se realizó un lavado externo de la cola del epidídimo con solución salina fisiológica ( $\mathrm{NaCl}$ al $0.9 \%)$ para remoción de la sangre y contaminantes externos. El tejido conjuntivo que recubre la cola se removió por disección, evitando cortar los vasos sanguíneos y el ducto epididimario. Se disecó la cola del epidídimo y se colocó en un vaso de precipitado de 100 $\mathrm{ml}$ conteniendo $4 \mathrm{ml}$ de dilutor base Tris precalentado $\left(35^{\circ} \mathrm{C}, \mathrm{pH} 6.78\right)$. El dilutor fue previamente preparado y almacenado en tubos Falcón de $15 \mathrm{ml}$, a $0{ }^{\circ} \mathrm{C}$ hasta su utilización. Los espermatozoides fueron recuperados por el método de picado en trozos con una tijera quirúrgica (Jone, 1978; Kaabi et al., 2003; Hori et al., 2005a,b) y mantenidos por 3 minutos a $35{ }^{\circ} \mathrm{C}$, permitiendo la salida de los espermatozoides. Cada suspensión de espermatozoides fue transferida a un tubo Falcón de $15 \mathrm{ml}$ estéril pre-calentado $\left(35^{\circ} \mathrm{C}\right)$.

Evaluación de espermatozoides epididimarios antes del proceso de criopreservación

Se evaluó la motilidad total y motilidad progresiva siguiendo métodos estándares (NAFA, 2002), y la integridad funcional de membrana plasmática mediante la prueba hipoosmótica (HOST) (Jeyendran et al., 1984). Se consideraron espermatozoides con membrana funcional $(\mathrm{HOST}+)$ los que reaccionaron al estrés hiposmótico mediante la 
Cuadro 1. Efecto del tiempo $(0,24,48$ y 72 horas) entre la muerte u orquiectomía de caninos hasta la recuperación de espermatozoides del epidídimo, sobre la calidad espermática antes y después del proceso de criopreservación (promedio \pm desviación estándar)

\begin{tabular}{|c|c|c|c|c|}
\hline \multirow{2}{*}{$\begin{array}{l}\text { Parámetros } \\
\text { evaluados }\end{array}$} & \multicolumn{4}{|c|}{ Grupo (tiempo de procesamiento) } \\
\hline & 0 & 24 & 48 & 72 \\
\hline \multicolumn{5}{|c|}{ Antes del proceso de criopreservación } \\
\hline $\begin{array}{l}\text { Motilidad total } \\
(\%)\end{array}$ & $79.5 \pm 4.97^{\mathrm{a}}$ & $69.0 \pm 8.43^{\mathrm{b}}$ & $66.5 \pm 7.09^{\mathrm{b}}$ & $52.0 \pm 13.17^{\mathrm{c}}$ \\
\hline $\begin{array}{l}\text { Motilidad progresiva } \\
(\%)\end{array}$ & $55.5 \pm 19.07^{\mathrm{a}}$ & $42.0 \pm 17.51^{\mathrm{ab}}$ & $45.0 \pm 11.55^{\mathrm{ab}}$ & $30.0 \pm 11.55^{\mathrm{b}}$ \\
\hline $\begin{array}{l}\text { Integridad funcional } \\
\text { de membrana }(\%)\end{array}$ & $76.43 \pm 4.0^{\mathrm{a}}$ & $64.13 \pm 13.51^{\mathrm{a}}$ & $69.42 \pm 12.11^{\mathrm{a}}$ & $68.92 \pm 10.35^{\circ}$ \\
\hline \multicolumn{5}{|c|}{ Después del proceso de criopreservación } \\
\hline $\begin{array}{l}\text { Motilidad total } \\
(\%)\end{array}$ & $17.00 \pm 7.64^{\mathrm{a}}$ & $13.70 \pm 3.97^{\mathrm{a}}$ & $11.40 \pm 6.10^{\mathrm{a}}$ & $5.20 \pm 4.21^{\mathrm{b}}$ \\
\hline $\begin{array}{l}\text { Motilidad progresiva } \\
(\%)\end{array}$ & $7.20 \pm 5.25^{\mathrm{a}}$ & $6.10 \pm 3.00^{\mathrm{a}}$ & $3.70 \pm 3.06^{\mathrm{ab}}$ & $1.80 \pm 1.55^{\mathrm{b}}$ \\
\hline $\begin{array}{l}\text { Integridad funcional } \\
\text { de membrana }(\%)\end{array}$ & $50.46 \pm 16.58^{a}$ & $48.67 \pm 12.74^{\mathrm{a}}$ & $48.90 \pm 25.03^{\mathrm{a}}$ & $47.02 \pm 15.16^{\circ}$ \\
\hline
\end{tabular}

a, b, c Superíndices diferentes dentro de filas indican diferencias significativas $(p<0.05)$

hinchazón de la parte distal de la cola espermática o enrollamiento de la misma, mientras que aquellos espermatozoides sin cambios en la cola se consideraron funcionalmente dañados (HOST -), y los resultados fueron expresados en porcentaje de espermatozoides con membrana funcional (HOST + .

\section{Criopreservación de espermatozoides epididimarios}

Para la congelación, $2 \mathrm{ml}$ de muestra diluida (Tris + espermatozoides epididimarios) fue colocada en un tubo Falcón de $15 \mathrm{ml}$ precalentado $\left(35^{\circ} \mathrm{C}\right)$. Se añadió $20 \%$ de yema de huevo (v/v). En esta suspensión se colocó, lentamente, y por las paredes del tubo, $5 \%$ de glicerol (v/v). La muestra fue someti- da a una curva de enfriamiento; para esto, el tubo falcón, que contiene la muestra, se colocó en un vaso de precipitado de $500 \mathrm{ml}$ conteniendo $250 \mathrm{ml}$ de agua natural pre-calentada $\left(35^{\circ} \mathrm{C}\right)$ y se llevó a refrigeración, de modo que la temperatura baje hasta $5^{\circ} \mathrm{C}$ en un tiempo, aproximado, de 90 minutos. El descenso de la temperatura fue de un grado centígrado cada 3 minutos.

La muestra refrigerada se envasó en pajillas de $0.5 \mathrm{ml}$. Las pajillas se colocaron en gradillas dentro de una canastilla previamente enfriada $5^{\circ} \mathrm{C}$, y esta se colocó en el cuello del tanque criogénico para ser expuesta a vapores de nitrógeno durante 20 minutos hasta alcanzar $-25^{\circ} \mathrm{C}$; luego se sumergió directamente en el nitrógeno líquido. 
Evaluación de espermatozoides epididimarios después del proceso de criopreservación

Para la descongelación de los espermatozoides, cada pajilla fue sumergida en agua a $40{ }^{\circ} \mathrm{C}$ por 40 segundos, y una alícuota fue colocada en un microvial pre-calentado a 35 ${ }^{\circ} \mathrm{C}$, e inmediatamente se evaluó la motilidad total, motilidad progresiva e integridad funcional de membrana.

\section{Análisis Estadístico}

Los datos fueron transformados a valores angulares (angulo $=$ arcoseno $\sqrt{x}$ ) para llevarlos a la distribución normal (Zar, 1999). Se empleó el análisis de varianza (ANOVA) para determinar diferencia estadística entre la motilidad espermática, integridad funcional de membrana, concentración y morfología espermática (variables dependientes) en el tiempo (0, 24, 48 y 72 horas) (variables independientes); y la prueba de Tukey para determinar diferencias entre grupos. Se trabajó con el programa estadístico Prism® v. 3.0.

\section{Resultados}

Los resultados obtenidos de la evaluación realizada antes y después del proceso de criopreservación se encuentran en el Cuadro 1. La motilidad espermática, tanto antes como después de la criopreservación se vieron afectadas por el tiempo transcurrido entre la muerte del animal o la orquiectomía y la recuperación de los espermatozoides $(p<0.05)$.

La motilidad total previa a la criopreservación disminuyó desde 79.5 a $52.0 \%$ luego de 72 horas de almacenamiento a $5^{\circ} \mathrm{C}$ $(\mathrm{p}<0.05)$. Una similar tendencia se presenta para la motilidad progresiva $(\mathrm{p}<0.05)$. Luego de la criopreservación, la disminución de la motilidad total como de la progresiva es igualmente significativa, aunque se parte de motilidades bastante bajas (17.0 y $7.2 \%$ en el Grupo 0).
Los valores de integridad funcional de membrana, antes y después de la criopreservación, no mostraron cambios de importancia durante el tiempo de almacenamiento.

\section{Discusión}

Los resultados de motilidad total, motilidad progresiva e integridad funcional de membrana muestran una tendencia a disminuir los valores con el incremento del tiempo entre la orquiectomía o muerte y la recuperación de los espermatozoides del epidídimo, siendo esta disminución significativa para los parámetros de motilidad total y progresiva.

La motilidad total antes de la criopreservación fue superior a la reportada por Tittarelli et al. (2006) y por Ponglowhapan et al. (2006), aunque ligeramente inferior a la encontrada por Hori et al. (2005a). De igual manera, los resultados de motilidad total encontrados después de la criopreservación fueron inferiores a los encontrados por Hori et al. (2005a) y Ponglowhapan et al. (2006). Los valores superiores de motilidad encontrados antes y después de la criopreservación por Hori et al. (2005a) se pudieron deber a la utilización de fluido prostático como medio para recuperar los espermatozoides del epidídimo, ya que puede contener algunas sustancias que pudieron haber protegido al espermatozoide en el proceso de criopreservación; sin embargo, la importancia funcional del plasma seminal aún es controversial. Rota et al. (1995) demostraron en caninos que el plasma seminal puede ser perjudicial para la motilidad espermática bajo ciertas condiciones de almacenamiento. En un trabajo reciente, Hori et al. (2009) obtuvieron mayores porcentajes de motilidad y viabilidad al descongelado en los espermatozoides epididimarios caninos recuperados con fluido prostático (FP) que con Tris-fructosacitrato y yema de huevo (EYT-FC), lo que sugiere que los espermatozoides epididimarios sensibilizados en PF son resistentes a la criopreservación. No obstante, se requieren 
estudios adicionales para dilucidar el efecto benéfico del plasma seminal en la obtención de una mejor calidad de espermatozoides epididimarios al descongelado.

El mayor porcentaje de motilidad total luego de la criopreservación obtenido por Ponglowhapan et al. (2006) pudo deberse a que después del descongelamiento, la pajilla fue vaciada en una solución Tris-fructosa citrato a $37^{\circ} \mathrm{C}$, lo cual pudo permitir la liberación de algunas sustancias esenciales utilizadas en la congelación de semen como el glicerol, y que algunos autores sugieren que ejerce efectos tóxicos en el espermatozoide (Curry, 2000; Holt, 2000).

El principal factor que produce una disminución en la motilidad de los espermatozoides epididimarios antes de la criopreservación, es el proceso de autolisis como consecuencia del daño celular causado por la hipoxia y anoxia que se produce por la detención del flujo de sangre hacia los tejidos del testículo y epidídimo después de la muerte del animal (Tittarelli et al., 2007). Otro factor importante que produce una disminución de la motilidad después de la criopreservación es el mismo proceso de criopreservación, ya que es una técnica que puede conducir a la muerte o bien a alteraciones funcionales del espermatozoide (Amann y Pickett, 1987).

El mantenimiento de la integridad funcional de la membrana plasmática es de fundamental importancia para que una célula espermática finalice su función primordial que es la fecundación; además, para recibir señales que modifican a la célula a lo largo del proceso de espermatogénesis, así como en el tránsito y almacenamiento en el epidídimo, y finalmente en la capacitación (Cunningham, 2003). Los resultados de integridad funcional de membrana antes y después de la criopreservación son similares a los encontrados por Ponglowhapan et al. (2006), donde tampoco se encontraron diferencias significativas; sin embargo, son ligeramente inferiores a los encontrados por Tittarelli et al
(2006). En el presente estudio, la membrana plasmática, a pesar de no ser afectada tan drásticamente como la motilidad, también se vio afectada por los procesos de autolisis y congelación.

\section{Conclusiones}

? La motilidad total y progresiva antes y después de la criopreservación se encuentra afectada en espermatozoides epididimarios recuperados a las 24 y 72 horas de almacenamiento a $5{ }^{\circ} \mathrm{C}$, mientras que la integridad funcional de membrana no se ve afectada dentro de las 72 horas de almacenamiento.

? Es posible obtener espermatozoides epididimarios funcionales hasta las 48 horas de la orquiectomía o muerte del canino, pudiendo ser congelados. Sin embargo, se obtiene una baja calidad espermática después de la criopreservación.

\section{Literatura Citada}

1. Amann RP, Griel LC. 1976. Fertility of bovine spermatozoa from rete testis, cauda epididymidis and ejaculated semen. J Dairy Science 57 (2): 212-219.

2. Amann RP, Pickett BW. 1987. Principles of cryopreservation and a review of cryopreservation of stallion spermatozoa. Equine Vet Sci 7: 145-176.

3. Blash S, Melican D, Gavin W. 2000. Criopreservation of epididymal sperm obtained at necropsy from goats. Theriogenology 54: 899-905.

4. Bruemmer JE, Reger H, Zibinski G, Squires EL. 2002. Effects of storage at $5{ }^{\circ} \mathrm{C}$ on the motility and cryopreservation of stallion epididymal spermatozoa. Theriogenology 58: 405-407.

5. Cunningham JG. 2003. Fisiología veterinaria. $3^{\mathrm{a}}$ ed. Madrid: Elsevier. $576 \mathrm{p}$.

6. Curry MR. 2000. Cryopreservation of semen from domestic livestock. Rev Reprod 5: 46-52. 
7. Hafez ESE. 2002. Preservación y criopreservación de gametos y embriones. En: Hafez ESE, Hafez B, eds. Reproducción e inseminación artificial en animales. $7^{\mathrm{a}}$ ed. México: MacGraw-Hill. p 441-452.

8. Hewitt DA, Leahy R, Sheldon IM, England GCW. 2001. Cryopreservation of epididymal dog sperm. Anim Reprod Sci 67: 101-111.

9. Holt WV. 2000. Fundamental aspects of sperm cryobiology: the importance of species and individual differences. Theriogenology 53: 47-58.

10. Hori T, Hagiuda K, Endo S, Hayama A, Kawakami E, Tsutsui T. 2005a. Unilateral intrauterine insemination with cryopreserved caudal epididymal sperm recovered from refrigerated canine epididymides. J Vet Med Sci 67: 1141-1147.

11. Hori T, Hagiuda K, Hayama A, Kawakami E, Tsutsui T. 2005b. Unilateral intrauterine insemination with prostatic fluid-sensitized frozen caudal epididymal sperm in beagles dogs. Theriogenology 63: 1573-1583.

12. Hori T, Uehara Y, Kawakami E, Tsutsui T. 2009. Influence of the time between removal and cooling of the canine epididymis on post-thaw caudal epididymal sperm quality. J Vet Med Sci 71: 811-815.

13. Ikeda H, Kikuchi K, Noguchi J, Takeda H, Shimada A, Mizokami T, Kaneko H. 2002. Effect of preincubation of criopreserved porcine epididymal sperm. Theriogenology 57: 1309-1318.

14. Jeyendran RS, Van Der Ven HH, Perez-Pelaez M, Crabo BG, Zaneveld LJ. 1984. Development of an assay to assess the functional integrity of the human sperm membrane and its relationship to other semen characteristics. J Reprod Fertil 70: 219-228.

15. Jone R. 1978. Comparative biochemistry of mammalian epididymal plasma. Comp Biochem Biophys 61B: 157-165.
16. Kaabi M, Paz P, Alvarez M, Anel E, Boixo JC, Rouissi H, Herraez P, Anel L. 2003. Effect of epididymis handling conditions on the quality of ram spermatozoa recovered post-mortem. Theriogenology 60: 1249-1259.

17. [NAFA] Nordic Association for Andrology. 2002. Manual on basic semen analysis. Sweden: NAFA y ESHRESIGA. 34 p.

18. Ponglowhapan S, Chatdarong K, Sirivaidyapong S, Lohachit C. 2006. Freezing of epididymal spermatozoa from dogs after cool storage for 2 or 4 days. Theriogenology 66: 1633-1636.

19. Rota A, Ström B, Linde-Forsberg C. 1995. Effects of seminal plasma and three extenders on canine semen stored at $4{ }^{\circ} \mathrm{C}$. Theriogenology 44: 885-900.

20. Tittarelli C, Savignone CA, Arnaudín E, Stornelli MC, Stornelli MA, De la Sota RL. 2006. Effect of storage media and storage time on survival of spermatozoa recovered from canine and feline epididymides. Theriogenology 66 : 1637-1640.

21. Tittarelli CM, Stornelli MC, Gimenez $F$, Savignone CA, De la Rota RL, Stornelli MA. 2007. Recuperación espermática epididimal como medio para preservar material genético. Rev Vet Cuyana 2: 16-20.

22. Yu I, Leibo SP. 2002. Recovery of motile, membrane-intact spermatozoa from canine epididymides stored for 8 days at $4{ }^{\circ} \mathrm{C}$. Theriogenology 57: 11791190.

23. Yu I, Songsasen N, Godke RA, Leibo SP. 2002. Differences among dogs in reponse of their spermatozoa to cryopreservation using various cooling and warming rates. Cryobiology 44: 62-78.

24. Zar J. 1999. Biostatistical analysis. $4^{\text {a }}$ ed. New Jersey: Prentice Hall. 929 p. 